\title{
Finite element analysis of mechanical stability of coarsened nanoporous gold
}

\author{
Hoon-Hwe Cho ${ }^{1}$, Yu-chen Karen Chen-Wiegart ${ }^{2}$ and David C. Dunand ${ }^{1 *}$ \\ ${ }^{1}$ Department of Materials Science and Engineering, Northwestern University, Evanston, \\ IL 60208, USA \\ ${ }^{2}$ National Synchrotron Light Source-II, Brookhaven National Laboratory, Upton, NY \\ 11973, USA \\ * Corresponding author: Prof. David C. Dunand \\ Email: dunand@northwestern.edu
}

\author{
1 \\ (C) 2016. This manuscript version is made available under the Elsevier user license \\ http://www.elsevier.com/open-access/userlicense/1.0/
}


The mechanical stability of nanoporous gold (np-Au) at various stages of thermal coarsening is studied via finite element analysis under volumetric compression using np$\mathrm{Au}$ architectures imaged via $\mathrm{x}$-ray nano-tomography. As the $\mathrm{np}-\mathrm{Au}$ is coarsened thermally over ligament sizes ranging from 185 to $465 \mathrm{~nm}$, the pore volume fraction is determinant for the mechanical stability of the coarsened $\mathrm{np}-\mathrm{Au}$, unlike the curvature and surface orientation of the ligaments. The computed Young's modulus and yield strength of the structures are compared with the Gibson-Ashby model. The geometry of the structures determines the locations where stress concentrations occur at the onset of yielding.

Keywords: coarsened nanoporous gold, mechanical stability, finite element (FE) analysis, volume fraction, surface characteristics 
Nanoporous gold (np-Au), an open-cell structure material with a bicontinuous structure of pores and ligaments at the nanoscale, has potential applications as sensor [1], actuator [2], catalyst [3, 4], battery electrode [5], and super-capacitor [6]. Open nanopore structures can be generated with characteristic length scales of 3-20 nm by controlling the dealloying conditions such as the applied potential, the dealloying time, and the electrolyte composition [7-10]. Thermal annealing coarsens the structures up to the micrometer scale [11-14], which changes the sizes of pore and ligament and the curvature and orientation of the ligament surfaces [14], thus affecting the mechanical, chemical and optical properties of coarsened np-Au [15]. In particular, understanding the mechanical behavior of the coarsened structures is important for device applications of np-Au. Most of current investigations studying mechanical properties of $\mathrm{np}-\mathrm{Au}$ involve experiments $[16,17]$, atomistic simulations [18, 19], and analytical modeling [20]. However, the role of continuum-mechanics simulation is crucial both to access regimes that are currently inaccessible experimentally and to predict the mechanical behavior of the coarsened npAu with length scales from nano- to micrometers.

In the present work, we study, via finite element (FE) analysis, the mechanical stability (defined as the resistance offered by a structure to irreversible processes such as plasticity, fracture or post-buckling adhesion) of the coarsened np-Au under compressive volumetric strain using experimental $\mathrm{np}$-Au architectures previously obtained from X-ray nano-tomography [14]. These structures have similar solid (and pore) volume fractions, but increasing feature sizes (e.g., ligament diameter and pore width), as well as different ligament surface characteristics (e.g., surface principal curvature distribution and surface morphological orientation), due to the non-self-similar coarsening behavior [14]. We compute the evolution, with increasing volumetric strain, of the maximum von Mises stress of the coarsened np-Au, which can be related to the local stress accumulation, and we compare the mechanical stability among the coarsened structures. We determine the Young's modulus and yield strength of the coarsened structures, which are compared with the Gibson-Ashby model. Finally, we report von Mises stress distributions of the coarsened structures at the onset of yielding.

A detailed description of the sample preparation and X-ray nano-tomography is given in Ref. [14]; the three coarsened structures used in the present work are dealloyed np-Au which was subsequently coarsened isothermally at $650{ }^{\circ} \mathrm{C}$ for 5,20 and $160 \mathrm{~min}$, times short enough so that no significant sintering is observed [14]. Mechanical properties of np-Au's ligament are assumed to be bulk-like [16, 17] and thus size-independent since the smallest ligament size in the coarsened structures is over $200 \mathrm{~nm}$, beyond the value where size effect on the mechanical behavior is not observed in the coarsened structures $[14,16]$.

The FE model is implemented in the ABAQUS/Standard software [21]. The volumetric compression is simulated in displacement control mode with a strain rate of $0.001 \mathrm{~s}^{-1}$. To apply volumetric compressive strain to the three structures, their outer surfaces are pinned while the temperature is increased without any change of mechanical properties, thus imposing equi-triaxial compressive strain, given the isotropic value of thermal expansion coefficient [22]. The three-dimensional (3D) meshes for the np-Au structures coarsened for 5, 20 and $160 \mathrm{~min}$ have 144,456, 147,955 and 106,640 tetrahedral elements, respectively, based on the experimental microstructures obtained from the X-ray nano-tomography [14], as seen in Fig. 1. For mesh quality and effective 
computation, each 3D mesh system with a volume of $3.25 \times 3.25 \times 3.25 \mu \mathrm{m}$ is cropped from the center region of the experimental microstructures [14].

For any given deformation condition, the accumulation of stress and strain at a localized region within the structure would cause the onset of concentrated deformation and load shedding, which would result in the architecture to be susceptible to undergoing an irreversible process such as plasticity and fracture. Fig. 2 (a) shows, for the three structures coarsened for 5, 20 and 160 min, the computed maximum von Mises and the average hydrostatic stresses plotted as a function of volumetric strain: the knee in the curves represents the strain at which global yielding occurs and stress only increases due to strain hardening, calculated from the data in Ref. [23] for bulk gold. The maximum stress in Fig. 2 (a) - taken as the average stress within the $10 \%$ of elements with the highest von Mises stress - is a measure of stress concentration and can be used as a criterion to determine mechanical stability of np-Au [22, 24]. The three coarsened structures have nearly identical local stress concentration evolution as a function of applied volumetric strain (Fig. 2(a)), even though the coarsened structures have ligaments with different surface principal curvature distribution (interfacial shape distribution) and surface morphological orientation (indication of facets) [14]. This indicates that the changes in surface curvature and orientation occurring during coarsening have negligible effect on the mechanical stability of np-Au.

As seen in Fig. 2 (a), the average hydrostatic stress evolution shows slight differences among the coarsened structures, which may be caused by differences in solid volume fraction which are 23.8, 24.9 and $26.3 \%$ for the cropped regions of the structures coarsened for 5, 20 and 160 min respectively. Fig. 2 (b) shows the detail of the transition region from elastic to plastic behavior in Fig. 2 (a). From Fig. 2 (b), the Young's modulus and yield strength (under triaxial compression) of the coarsened structures can be obtained using (i) the slope in the elastic region (bulk modulus) with the linear elasticity conversion formula for elastic modulus, and (ii) the onset of the plateau region upon yielding. There is a near-linear relationship between the solid volume fractions (from 23.8 to $26.3 \%$ ) and the Young's modulus and yield strength.

According to the standard model of mechanical properties of foam proposed by Gibson and Ashby, the relationship between the foam mechanical properties (Young's modulus $\left(E^{*}\right)$ and yield strength $\left.\left(\sigma^{*}\right)\right)$ and the relative density $\left(\frac{\rho^{*}}{\rho_{s}}\right)$ can be described as follows [25]:

$$
\begin{aligned}
E^{*} & =E_{s}\left(\frac{\rho^{*}}{\rho_{s}}\right)^{2} \\
\sigma^{*} & =0.3 \sigma_{s}\left(\frac{\rho^{*}}{\rho_{s}}\right)^{3 / 2}
\end{aligned}
$$

where the superscript $*$ and subscript s denote foam and bulk properties, respectively. Using the bulk properties of gold from Biener et al. and Mathur et al. $\left(E_{s}: 79 \mathrm{GPa}\right.$ and $\sigma_{s}$ : $80 \mathrm{MPa}[16,26])$, the Gibson-Ashby model predictions are compared with the results obtained from the FE model, as seen in Fig. 3. The Young's modulus and yield strength of the coarsened $\mathrm{np}-\mathrm{Au}$ increase with increasing Au volume fraction, as expected. The values from the FE model have steeper slope than the Gibson and Ashby model, which might be caused by the complex ligament shape, size and orientation of each coarsened structure. Nevertheless, Fig. 3 shows that the mechanical properties are broadly consistent for a narrow range of solid volume fraction when comparing the two models. 
This is despite the large difference between (i) the Gibson-Ashby model which is based on strut bending (for the stiffness) and strut plastic hinging (for yield) in an idealized, regular scaffold structure subjected to uniaxial strain, and (ii) the FEM calculations, which consider an irregular scaffold geometry subjected to equi-triaxial deformation, with stiffness given by the average elastic response of the struts subjected to a complex multi-axial elastic strain, and yield occurring at strut necks where stress concentrations exist, under triaxial deformation.

Fig. 4 shows the predicted von Mises stress distributions of the three np-Au structures at the onset of yielding, at an applied volumetric strain of $1.35 \times 10^{-3}$. At the onset of yielding, only $3 \%$ of all the elements of the three studied structures have yielded, i.e., show a von Mises stress in excess of the uniaxial yield strength of bulk gold (80 MPa [16]). In the structures coarsened for 5 and $20 \mathrm{~min}$, the stress is concentrated at the ligament necks, except for the outer surfaces subjected to the boundary condition, while the fully coarsened structure $(160 \mathrm{~min})$ does not have clearly defined the necks connecting with ligaments. The evolution with applied strain of the maximum von Mises stress are nearly the same for the three structures as seen in Fig. 2 (a), though there is a significant difference in strut geometry of each coarsened structure, demonstrating that the surface structure evolution during coarsening does not appear to significantly affect the mechanical stability of the coarsened np-Au.

In summary, the mechanical stability of coarsened $\mathrm{np}-\mathrm{Au}$ was investigated by carrying out a FE analysis of experimental structures (as previously imaged via nanotomography) subjected to volumetric compression. Despite variations of ligament size and surface characteristics among the np-Au foams coarsened to different times, a nearly identical local stress accumulation evolution is observed. However, geometrical differences among the coarsened structures affect the location where stress concentration occurs at the onset of yielding. The volume fraction of solid $\mathrm{Au}$ is confirmed to be an important parameter to determine the mechanical stability of the coarsened np-Au. The FE model determines the macroscopic mechanical properties such as Young's modulus and yield strength of the coarsened np-Au: the FE results from the complex architecture of np-Au (consisting of ligaments with wide spatial variations in diameter and orientation) under equi-triaxial strain are broadly consistent with the predictions of the simple, closedform Gibson-Ashby model for regular cellular structures with uniform struts under uniaxial strain. This work illustrates the importance of continuum-mechanics simulation which can predict the mechanical behavior of the coarsened $\mathrm{np}-\mathrm{Au}$ with length scale from nano- to micrometers. This work is also the first step towards understanding, using FE modeling, the mechanics of sub-microporous structures whose mechanical testing is currently inaccessible experimentally under complex stress state (e.g. triaxial compression).

\section{Acknowledgements}

This research was supported by the Dongbu Cultural Foundation. The X-ray nanotomography data were originally collected at Advanced Photon Source (APS) as detailed in Ref. [14]. Use of APS is supported by the US Department of Energy, Office of Science, Office of Basic Energy Sciences, under contract no. DE-AC02-06CH11357.

\section{References}


[1] H.J. Qiu, L.Y. Xue, G.L. Ji, G.P. Zhou, X.R. Huang, Y.B. Qu, P.J. Gao, Biosens Bioelectron, 24 (2009) 3014-3018.

[2] J. Biener, A. Wittstock, L.A. Zepeda-Ruiz, M.M. Biener, V. Zielasek, D. Kramer, R.N. Viswanath, J. Weissmuller, M. Baumer, A.V. Hamza, Nat Mater, 8 (2009) 47-51.

[3] C. Xu, X. Xu, J. Su, Y. Ding, J Catal, 252 (2007) 243-248.

[4] A. Wittstock, V. Zielasek, J. Biener, C.M. Friend, M. Baumer, Science, 327 (2010) 319-322.

[5] Y. Yu, L. Gu, X.Y. Lang, C.B. Zhu, T. Fujita, M.W. Chen, J. Maier, Adv Mater, 23 (2011) 2443-+.

[6] X.Y. Lang, A. Hirata, T. Fujita, M.W. Chen, Nat Nanotechnol, 6 (2011) 232-236.

[7] J. Weissmuller, R.C. Newman, H.J. Jin, A.M. Hodge, J.W. Kysar, Mrs Bull, 34 (2009) 577-586.

[8] Y. Ding, Y.J. Kim, J. Erlebacher, Adv Mater, 16 (2004) 1897-+.

[9] L.H. Qian, M.W. Chen, Appl Phys Lett, 91 (2007).

[10] B.C. Tappan, S.A. Steiner, E.P. Luther, Angew Chem Int Edit, 49 (2010) 4544-4565.

[11] F. Kertis, J. Snyder, L. Govada, S. Khurshid, N. Chayen, J. Erlebacher, Jom-Us, 62 (2010) 50-56.

[12] M.E. Cox, D.C. Dunand, Mat Sci Eng a-Struct, 528 (2011) 2401-2406.

[13] E. Seker, J.T. Gaskins, H. Bart-Smith, J. Zhu, M.L. Reed, G. Zangari, R. Kelly, M.R. Begley, Acta Mater, 55 (2007) 4593-4602.

[14] Y.C.K. Chen-Wiegart, S. Wang, Y.S. Chu, W.J. Liu, I. McNulty, P.W. Voorhees, D.C. Dunand, Acta Mater, 60 (2012) 4972-4981.

[15] S.O. Kucheyev, J.R. Hayes, J. Biener, T. Huser, C.E. Talley, A.V. Hamza, Appl Phys Lett, 89 (2006).

[16] J. Biener, A.M. Hodge, J.R. Hayes, C.A. Volkert, L.A. Zepeda-Ruiz, A.V. Hamza, F.F. Abraham, Nano Lett, 6 (2006) 2379-2382.

[17] A.M. Hodge, J.R. Hayes, J.A. Caro, J. Biener, A.V. Hamza, Adv Eng Mater, 8 (2006) 853-857.

[18] D. Farkas, A. Caro, E. Bringa, D. Crowson, Acta Mater, 61 (2013) 3249-3256.

[19] J.F. Rodriguez-Nieva, C.J. Ruestes, Y. Tang, E.M. Bringa, Acta Mater, 80 (2014) 67-76.

[20] G. Pia, F. Delogu, Acta Mater, 99 (2015) 29-38.

[21] H.D. Hibbitt, Nucl Eng Des, 77 (1984) 271-297.

[22] H.H. Cho, Y. Cho, H.N. Han, Acta Mater, 97 (2015) 199-206.

[23] T. Suzuki, A. Vinogradov, S. Hashimoto, Mater Trans, 45 (2004) 2200-2208.

[24] Y. Cho, T.H. Ahn, H.H. Cho, J.H. Shin, J.H. Moon, S. Yang, I.S. Choi, H.N. Han, J. Li, Model Simul Mater Sc, 21 (2013).

[25] L.J. Gibson, M.F. Ashby, Cellular solids : structure and properties, 2nd ed., Cambridge University Press, Cambridge ; New York, 1997.

[26] A. Mathur, J. Erlebacher, Appl Phys Lett, 90 (2007). 


\section{Figures}

(a)

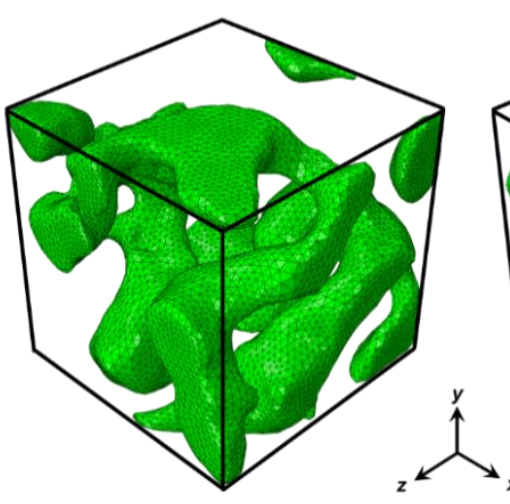

(b)

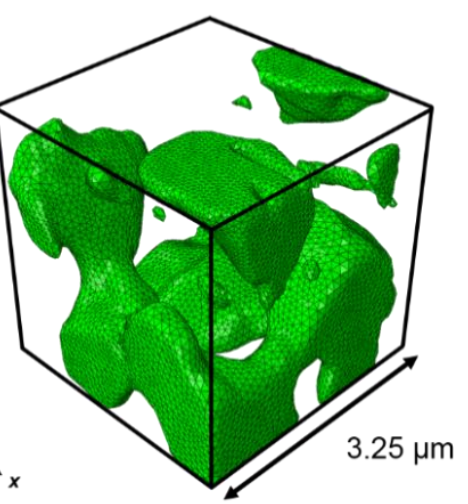

(c)

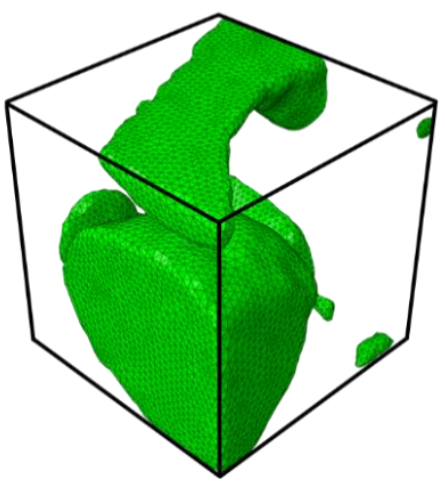

(240 words)

Figure 1 - 3D meshes for np-Au structures coarsened at $650{ }^{\circ} \mathrm{C}$ for (a) 5, (b) 20 and (c) $160 \mathrm{~min}$, as measured by nano-tomography [14]. 
(a)

(b)

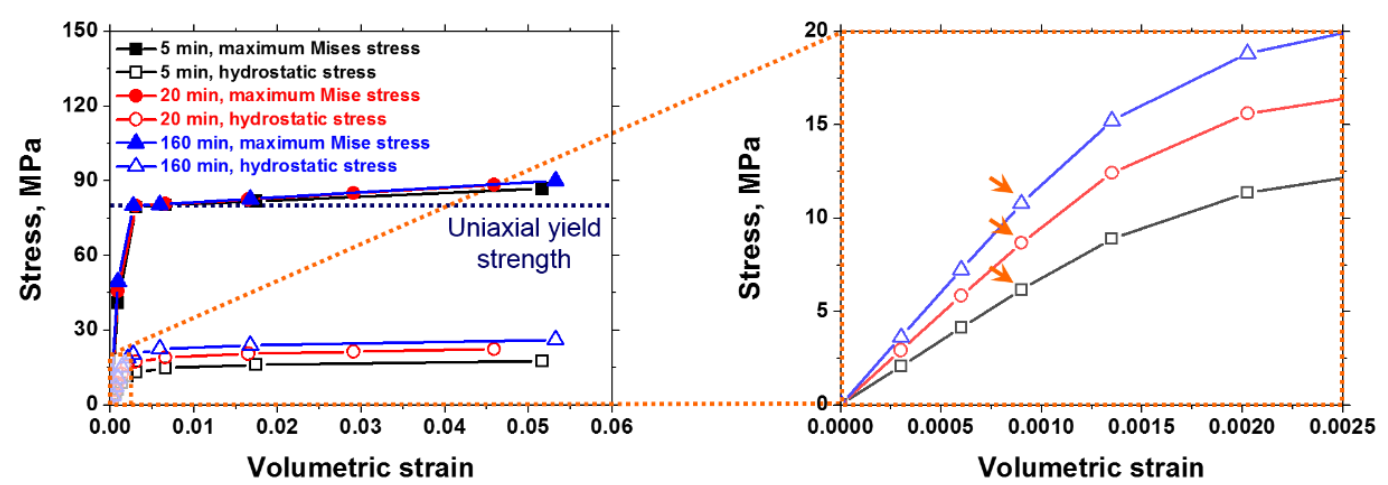

(240 words)

Figure 2 - (a) Evolution with applied volumetric strain of maximum von Mises stress (averaged over the $10 \%$ of elements with highest von Mises value) and hydrostatic stress (averaged over all elements, and plotted as positive value) for np-Au structures coarsened for 5, 20 and $160 \mathrm{~min}$, and (b) detailed view of the transition region from elastic to plastic behavior of Fig. 2 (a) for hydrostatic stress. Solid and hollow symbols indicate maximum von Mises and hydrostatic stresses, respectively. The hydrostatic stresses have negative values, indicating that the structures are under volumetric compression. Horizontal dotted line in (a) corresponds to value of uniaxial yield strength of bulk gold. Arrows in (b) correspond to values of yield strength reported in Fig. 3. 


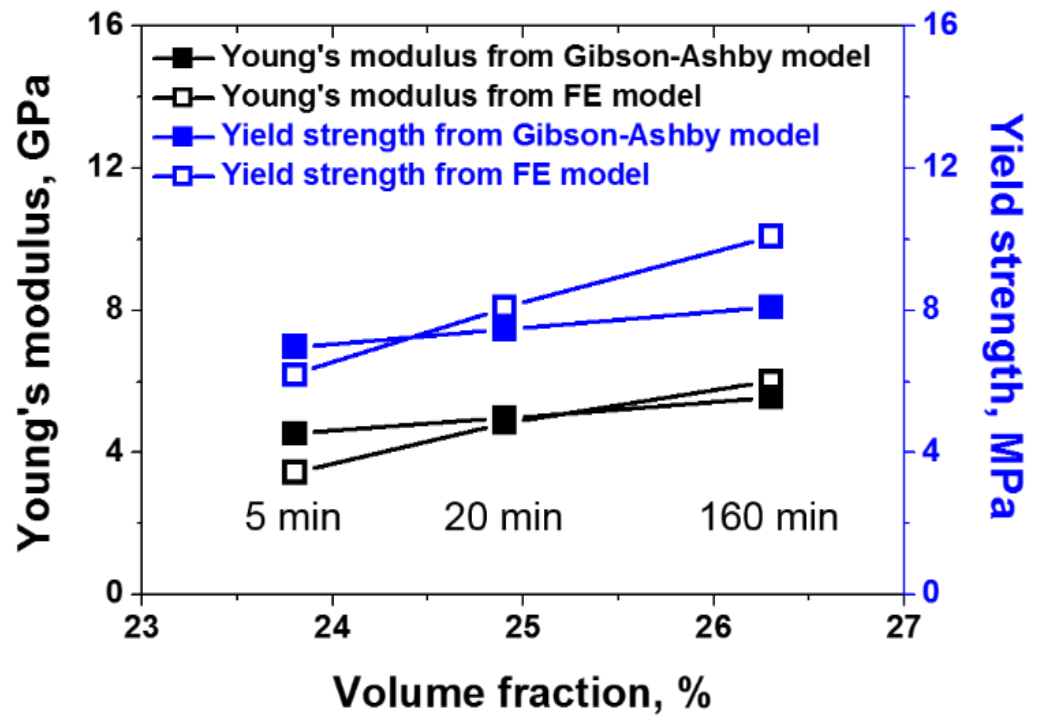

(320 words)

Figure 3 - Comparison, as a function of solid volume fraction, of Young's modulus and uniaxial yield strength obtained from the Gibson-Ashby model (Eqs. 1-2) and Young's modulus and triaxial yield strength from FE models for the three coarsened np-Au structures. 
(a)

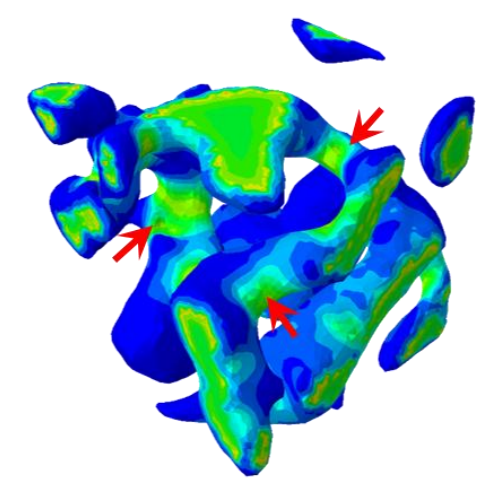

(c)

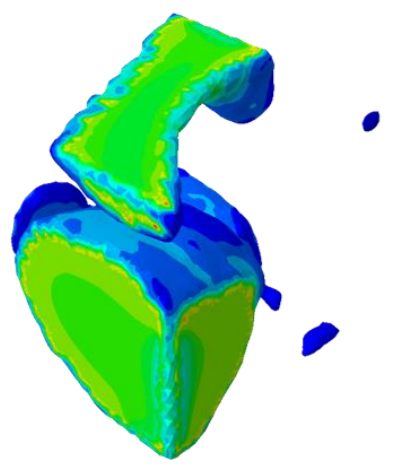

(b)

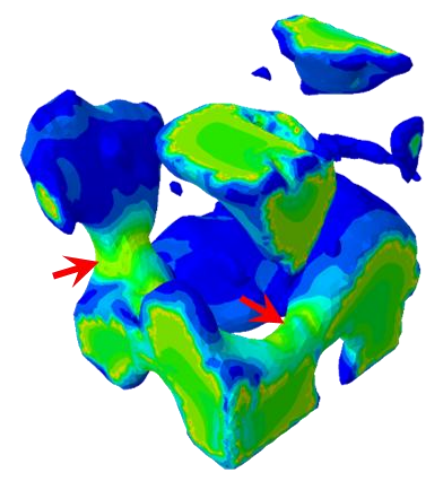

Stress, MPa

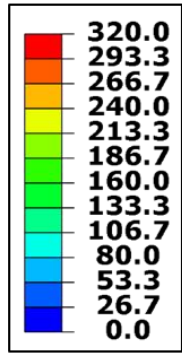

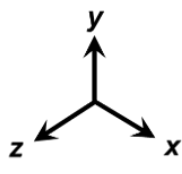

(480 words)

Figure 4 - Calculated von Mises stress distributions of actual np-Au structures coarsened for (a) 5, (b) 20 and (c) $160 \mathrm{~min}$ at the onset of yielding, showing stress concentrations. Arrows in (a) and (b) point to stress concentrations at ligament necks. 

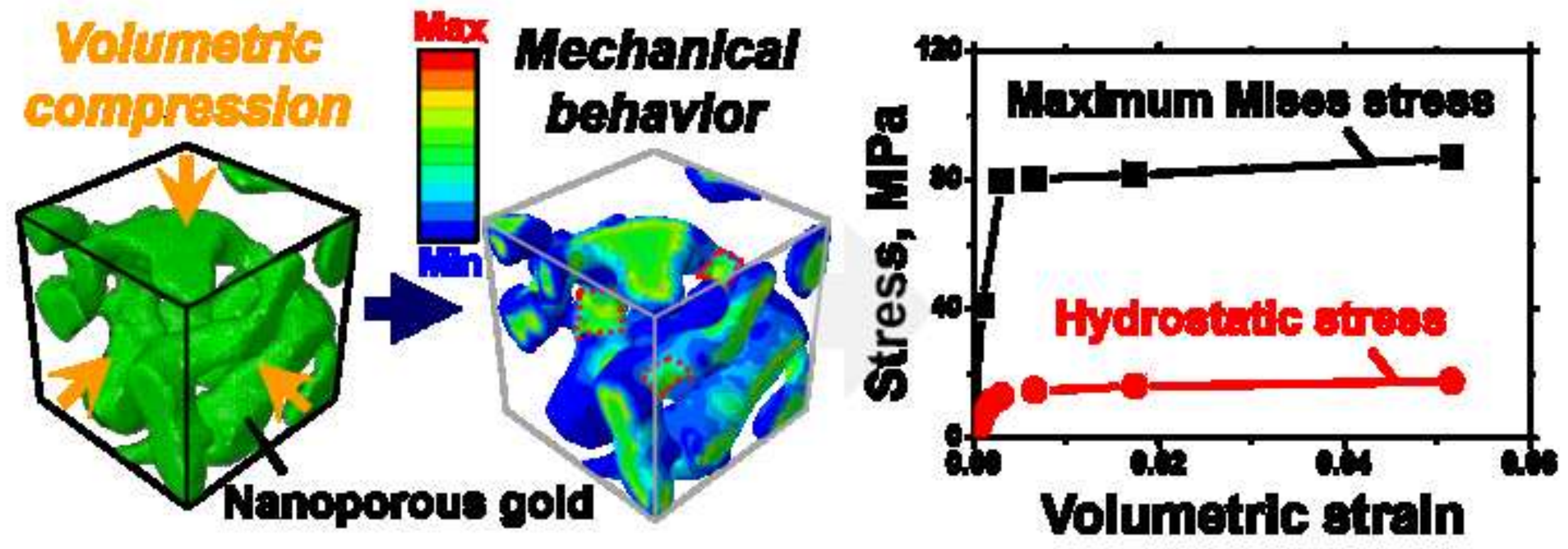